\title{
O Que a Epidemiologia Pode Ainda Fazer de Relevante?
}

\footnotetext{
- Psicologa da Asfoc/ Fiocruz. - Médico do Setor de Psicologia e Psiguiatria do $H$. Evandro Chagas/ F iocruz.

* * Professor Adjunto da Ensp/
} Fiocruz.

\author{
Marisa de Souza Cardim* \\ Belarmino Alves de Azevedo ** \\ Anastácio Ferreira Morgado ***
}

Trata-se de uma sucinta revisão de alguns aspectos da epidemiologia ainda mal conhecidos, - por serem polêmicos ou cujo desenvolvimento é recente ou apenas se esboça. Enquanto método, haveria duas epidemiologias: uma em que se esmera cada vez mais no rigor metodológico e análise sofisticada dos dados em computador; a outra contenta-se com métodos simples, alia-se fortemente com as ciências sociais, a ecônomia e as ciências do comportamento, - com o objetivo de achar soluções para os problemas de saúde. A importância das conseqüências dos fatores sócio-econômicos sobre a saúde foi avaliada a partir de publicações das pesquisas epidemiológica e clĺnica; aquela importância aumentou no primeiro tipo de pesquisa e tende a desaparecer no segundo. A questão de avaliar o estado de saúde de populações ficou sem avançar, pois, sem operacionalizar indicadores de saúde positiva, de fato avalia-se estado de doença. Enfim, reconheceu-se o advento algo original de um desdobramento promissor da epidemiologia: o estudo das representações da Antropologia, tais como mitos, crenças, comportamento e instituiçōes.

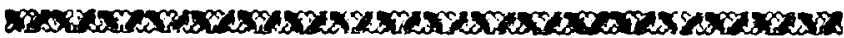

\section{VARIAÇÃO EM TORNO DO MÉTODO}

O modelo base de análise em epidemiologia fundase na estatística, mas a sua classificaçāo enraíza-se na patologia. Estuda-se o fenômeno de saúde representado por uma ou mais variáveis: dependentes e explicativas, consideradas relevantes para um dado problema de saúde. Os métodos estatísticos empregados permitem, segundo a etapa do trabalho epidemiológico, colocar em evidência uma associação entre variáveis e o fenômeno de saúde, mas raramente chega a afirmar uma associação causal. $\mathrm{Na}$ maioria das vezes esses estudos se restringem a descrever a população estudada e a "grupos de risco".

Cadernos de Satde Pública, RJ, 7(1):5, jan/mar, 1991. 
Este "modelo dominante" desenvolvido pelos epidemiologistas foi evoluindo e sendo progressivamente adaptado, à medida que a epidemiologia expandiu seu domínio de estudo. $\mathrm{O}$ modelo inicial, expresso em termos probabilísticos, tem sido transportado também para as doenças não-transmissíveis, como também no estudo dos fatores sócio-econômicos de desenvolvimento social sem atrelá-lo a doenças. A estatística

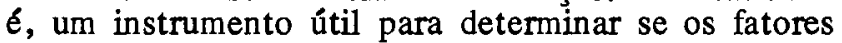
ocorreram por acaso; muitas vezes os resultados não são significativos apenas devido ao tamanho da amostra e, com isto, boas teorias são eliminadas.

No seio do método epidemiológico, constata-se facilmente um fenômeno ao mesmo tempo vantajoso e preocupante. De certa forma, a pesquisa nesta área é,cada vez mais, realizada com "duas epidemiologias". Uma destas refina-se no rigor formal dos tipos de estudos epidemiológicos (descritivo, caso-controle e coorte) e na análise estatística. A "outra epidemiologia" é mais flexível no formal e, arrojadamente, tenta articular-se com as demais disciplinas voltadas para o processo saúde-doença na população. A perspectiva desta epidemiologia é de um grande desafio para os que nela trabalham. Em suma, os epidemiologistas precisam estudar outras áreas, enquanto economistas, sociólogos, antropólogos, administradores de serviço etc, precisam aprender epidemiologia. De um lado isto é estimulante, mas, de outro lado, comportam-se riscos de improvisaçōes apressadas e até banalizaçōes de um ou outro ramo da ciência.

Entre as "duas epidemiologias" já há conflitos que, embora menores do que os existentes entre os clínicos e epidemiologistas, são suficientemente importantes para serem mencionados. Um bom indicador $\varepsilon$ o da dispersão das pesquisas publicadas pelos epidemiologistas não-ortodoxos em um elenco de revistas - de saúde pública, medicina social, de serviços populacionais, de antropologia etc.

$\mathrm{Na}$ busca do rigor, sobretudo no método de seleção de variáveis, por exemplo, de fatores de risco num dado grupo de pessoas, pode-se eclipsar o objeto e, conseqũentemente, não mostrar de fato o problema na população. Essa é uma clássica falha epistêmica, onde se superestima o valor do instrumento, sem atentar para a soberania do objeto e o raciocínio investigador (raisonnement, reasoning). Não somos os únicos a ter essa opinião; por exemplo, por reconhecer a enorme importância de um particular raciocínio, aproximando-se aos cânones de J.S. Mill em muitos pontos da obra de Freud, que Castiel (1) identificou o pai da psicanálise como sendo, também, um epidemiologista. Sawyer (5) focaliza a potencial contribuição da pesquisa social para o controle da Malária, com 
a inclusão de várias disciplinas de ciências sociais; criticou com propriedade a análise epidemiológica existente, a qual busca soluçōes puramente técnicas para um problema social. Citando o exemplo do controle da Malária na Regiāo Amazônica, onde a doença tem aumentado em $1.500 \%$, mostra que o ambiente físico e social dessa região requer diversificaçăo das estratégias de controle existentes.

Alguns problemas populacionais como a migração-emigração, a violência, o estresse e os transtomos mentais têm desafiado a pesquisa populacional há algumas décadas. O estresse ilustra bem isso, porque empiricamente ninguém duvida de seu papel em acarretar, no mínimo, grande desconforto pessoal e conflitos interpessoais. Embora os estudiosos em psicossomática e psicossociologia sejam convictos de que o estresse seja um fator causal importante de muitas afecçöes, a maior parte dos estudos têm se revelado não convincentes para estabelecer tal associaçāo. Entretanto, em condiçốes extremas de estresse, a verdade da associaçāo foi demonstrada, como no estudo de Reid (4) entre os aviadores combatentes da Royal Air Force, na Segunda Guerra Mundial. Uma parte de seus resultados encontra-se na tabela $I$, de onde se destacam as seguintes observaçōes:

1 - Desenvolver "neurose" antes do embarque no avião ou durante a operação de bombardeio correlacionou-se significativamente com "ansiedade" decorrente de:

a) risco de morrer, indicado pelas taxas de baixas (mortes) de aviadores nos bombardeios precedentes;

b) taxas de desaparecidos durante as missōes;

c) taxas de novatos na tripulação, ou seja, que a inexperiência no embarque aumenta o estresse.

2 - Desenvolver "neurose" nẫo se correlacionou significativamente com as medidas de esforço nas operaçōes de bombardeio.

$\mathrm{O}$ estudo de Reid e a experiência do dia-a-dia de cada um indicam, pois, que o estresse é morbigênico. É provável, então, que os resultados pouco convincentes das pesquisas sobre estresse decorram de falhas no desenho ou na abordagem metodológica dos estudos.

\section{REDE DE CAUSALIDADE}

Atualmente discute-se muito sobre a multicausalidade de uma doença, porém os estudos realizados pelos investigadores demonstram que $\varepsilon$ mais uma retórica discursiva, porque neles buscam-se uma ou poucas

Cadernces de Satde Pública, RJ, 7(1):6-16, jan/mar, 1991. 
causas para a doença. De fato não se tenta compreender a doença como resultado da interação de fatores que atuam dentro de uma convincente "rede de causalidade". Lembrando-se do marco conceitual da epidemiologia da tríade agente-hospedeiro-ambiente, se não se pensa nela, esquecem-se dos fatores atuantes no hospedeiro e no ambiente.

No modelo da rede de causalidade, freqüentemente não se levam em conta os fatores sócio-econômicos, isto $\mathcal{E}$, estes não são apreendidos no processo gerador do dano. Esta abordagem macroscópica da influência da organizaçāo econômica e social foi tentada por alguns autores, citados por Goldberg (3), os quais integraram diversas variáveis, representando diretamente o impacto do sistema social sobre o dano na população. Densidade populacional, a população ativa nos diversos setores da economia, a produção e consumo de certos bens, a densidade de serviços médicos etc foram as variáveis "macroscópicas" escolhidas por aqueles autores. Os resultados obtidos por eles mostram que, em geral, estas variáveis macroscópicas possuem um poder explicativo muito importante. Podemos lembrar a bem evidenciada ligação entre Produto Nacional Bruto e mortalidade geral ou mortalidade por tuberculose. Claro que năo incluir as variáveis macroscópicas na categoria dos fatores independentes significa podar os galhos mais robustos da rede de causalidade. Desenvolver indicadores operacionais dessas variáveis é um desafio que se coloca para os epidemiologistas que se preocupam em achar soluçōes para os problemas de saúde.

\section{TABELA I}

Coeficientes de Correlação (R) entre Fatores Operacionais em Vôos de Bombardeio da RAF e Taxas de "Neurose" (Síncope, Nervosidade e Sintomas Digestivos) em Posteriores Operaçōes de Bombardeio

\begin{tabular}{|c|c|c|c|}
\hline Grupas de Fatores de Risco & (R) & Fatores de Esforço em Combate & (R) \\
\hline $\begin{array}{l}\text { Taxas de baixas nas operaçöes } \\
\text { no mesmo mEs } \\
\text { no mEs anterior } \\
\text { Taxas de desaparecidos em missöes } \\
\text { no mesmo més } \\
\text { no mEs anterior } \\
\text { Taxas de aviadores novatos } \\
\text { no mesmo més } \\
\text { no mEs anterior }\end{array}$ & $\begin{array}{l}\bullet 42^{* *} \\
-33^{* *} \\
.24^{*} \\
.24^{*} \\
.29^{*} \\
.28^{*}\end{array}$ & 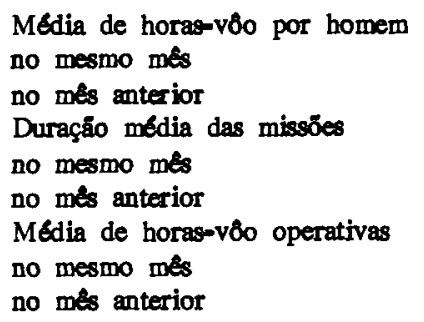 & $\begin{array}{l}\cdot 12^{\mathrm{ns}} \\
.12^{\mathrm{ns}} \\
.04^{\mathrm{ns}} \\
.09^{\mathrm{ns}} \\
.03^{\mathrm{ns}} \\
.03^{\mathrm{ns}}\end{array}$ \\
\hline
\end{tabular}

*P<05; * $\mathrm{p}<01$

Fonte: Adaptado de Reid (1948)

Cadernos de Salide Publica, RJ, 7(1):6-16, jan/mar, 1991. 

PROCESSO SAÚDE-DOENÇA

As taxas de mortalidade têm sido tradicionalmente o indicador mais usado para diagnosticar a situação de saúde, porque é fácil calculá-las, por razōes conceituais (a morte não se presta a ambigüidade) e funcional: preocupação com grupos específicos, como mortes prematuras na comunidade, em parturientes etc.

$\mathrm{O}$ indicador de morbidade também tem sido muito utilizado, mas sem atentar-se devidamente para o fato de que o conceito de caso seja extremamente variável tanto geográfica quanto cronologicamente. Embora úteis, estes dois indicadores são insuficientes e limitados; eles não refletem o peso dos problemas de saúde, seja ao nível individual ou coletivo. Por exemplo, as taxas de incidência e prevalência são calculadas com base no número de casos de doenças ou de anomalias congênitas, não permitindo diferenciar, para uma mes* ma doença, os casos que se acompanham de perturbações funcionais, que impedem a atividade habitual do doente, daqueles que são compatíveis com um prosseguimento destas atividades.

Os indicadores de morbidade repousam sobre as observações individuais, as quais são em seguida agregadas, de forma a fornecer uma medida da doença de um grupo ou de uma população. Vários autores, principalmente sociólogos, criticam estes indicadores e assinalam o fato de que os problemas de saúde que aparecem num grupo ou população não são simplesmente a soma dos problemas individuais que ai se encontram. Nas comunidades existem problemas de saúde específicos por suas ligações sociais, institucionais, geográficas etc como também a definição de saúde já possui natureza social e psicológica. Goldberg (3) insiste que não existe definição operacional das mer'idas de morbidade de uma população que integrem as dimensões sociológica e psicológica.

Dentro dessas limitações, toma-se muito difícil evidenciar a relação entre doença e situação sócio-econômica; esta fica somente como um fator de ação indireta, expressando-se de maneira polimorfa e não- específica que acentua ou atenua certas manifestaçōes da doença. Avaliar o efeito dos fatores sócio-econômicos sobre a saúde através da quantificação das doenças e de óbitos redunda em uma estimativa parcial $\mathrm{e}$ infiel daquele efeito.

Ocorrem numerosas tentativas de se operacionalizar o estado de saúde, isto é, de como decidir que tal estado de saúde de uma população é melhor que o de outra população. Tais tentativas foram bastante criticadas por sua subjetividade, com, conseqüente- 
mente, resultados inconsistentes. A principal crítica provém do fato de que uma tal comparação levanta necessariamente o conceito de saúde positiva. Essa noção constitui a evidência de maior impacto, de que os chamados "indicadores de saúde" avaliam apenas uma parte, a partir da qual pretende-se avaliar o todo da saúde. Se ampliadamente empregados, estes indicadores incluem os "riscos sociais" (por exemplo, estresse ocupacional, conflitos na família), além das taxas de mortaljdade e morbidade. De fato, há populaçōes semelhantes nesses indicadores, mas que diferem muito quanto a níveis de bem-estar social, satisfação obtida no emprego, tempo que é reservado para prática de lazer e genuíno sentimento de utilidade no meio em que vive. Todo o problema aqui é definir esses conceitos e, a partir deles, operacionalizar indicadores. Essa é uma tarefa muito difícil, pois muitas variáveis importantes de saúde positiva são de natureza subjetiva. Para avaliar isso de fato, só há um meio: basear-se no que $o$ indivíduo relata sobre ele próprio. $E$ isso levanta a questão da confiabilidade do relato verbal de categorias do sujeito, sem contar com nenhum elemento de variação através de um referencial externo ao sujeito. Entretanto, se é ciência de boa qualidade ou năo, seria um absurdo desprezarem-se os dados de relato verbal por uma não-credibilidade colocada a priori. Embora seja louvável a tendência a desenvolver indicadores objetivos de saúde positiva, ocorrerá necessariamente uma grande limitação, se a dimensão subjetiva não for levada em conta. Os caminhos abertos na direção das pesquisas que examinem a saúde positiva são:

a) alargar o campo da saúde, introduzindo-se novas dimensōes positivas;

b) propor novos meios de medir os fenômenos que não eram vistos pelos métodos anteriores;

c) abolição do monopólio médico sobre a apreciação dos problemas de saúde e,

d) o indivíduo isolado ou em grupo como fonte de referência idônea de julgamento.

\section{ESTUDOS DE EPIDEMIOLOGIA SOCIAL}

Alguns autores preocuparam-se em avaliar a importância dos determinantes sócio-econômicos da saúde, dentre os quais citamos o estudo de Vincent, citado por Goldberg (3) e Fletcher (2). Vincent estudou a evolução do interesse dado pelos epidemiologistas aos fatores sócio-econômicos, a partir dos abstracts publicados de 1965 a 1976 pela National Library of American Medicine. $\mathrm{O}$ estudo de Fletcher e Fletcher foi voltado para os artigos originais, relatando os resultados de trabalhos de pesquisa clínica publicados de 


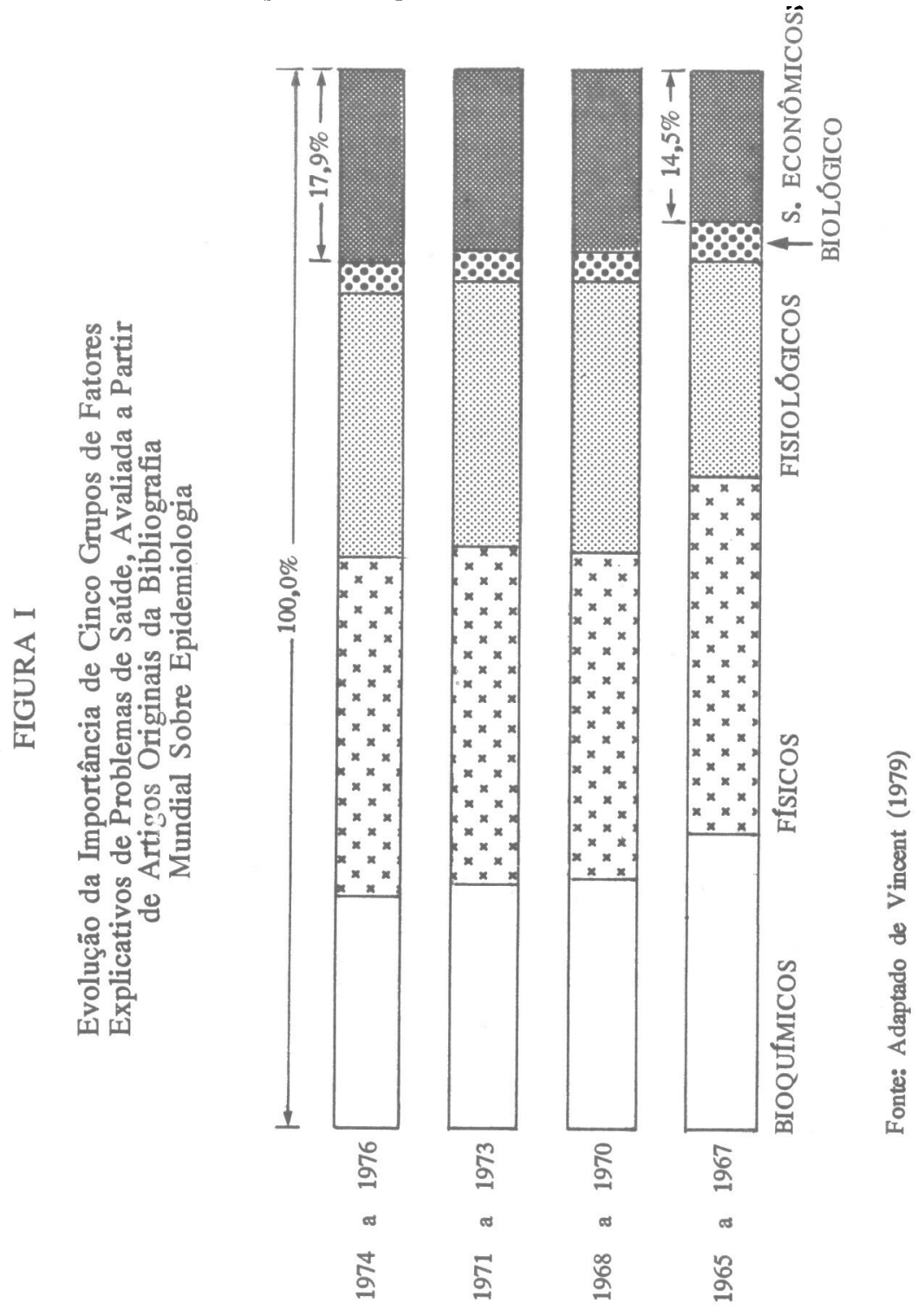

1946 a 1976 em três grandes revistas anglosaxônicas (Journal of the American Medical Association, New England Journal of Medicine $e$ Lancet). O essencial dos dois referidos estudos pode ser visualizado e comparado mediante os gráficos I e II.

Embora o método e o contexto dos trabalhos de Vincent e de Fletcher e Fletcher sejam diferentes, o fenômeno estudado por eles é o mesmo - e precisamente por isso referentes às perspectivas da pesquisa em Epidemiologia social. 
Pela comparação, destacamos as seguintes observações:

1 - Comum aos dois estudos é o pequeno valor concedido aos fatores sócio-econômicos na geração do processo saúde-doença.

2 - Nos artigos oriundos de pesquisas epidemiológicas, o valor daqueles fatores foi constantemente crescente nos sucessivos triênios estudados. Em bora não se formeça teste estatístico para as diferenças, é provável que estas sejam significativas, pois o tamanho da amostra foi grande.

3 - Nos estudos realizados por clínicos, o valor daque les fatores foi muito pequeno e, ainda mais importante que isto, o referido valor foi se reduzindo esmagadoramente, ao longo das décadas estudadas.

É importante acrescentar que, no estudo de Fletcher e Fletcher, uma outra variável tão importante quanto a sócio-econômica - o estado emocional dos pacientes - foi praticamente desprezada, com o corre: das décadas, a partir de 1956. Por outro lado, estes autores encontraram um progressivo aumento da importância de variáveis nitidamente tecnológicas, exames de laboratório, por exemplo, que foram, respectivamente, de $79,0 \%, 80,0 \%$ e $90,0 \%$ nas mencionadas décadas.

O que se pode comentar a partir dessas observações? As razões para tais resultados podem ser principalmente atribuídas à posição secundária da pesquisa em Saúde Pública e das disciplinas diretamente envol. vidas no seu campo: Sociologia e Economia da Saúde, Epidemiologia. Na pesquisa clínica, o reducionismo à biologia pode também ter sido importante, o que ocorre freqüentemente hoje em dia. A este respeito pode-se citar o exemplo da pesquisa em doenças mentais, que tem se restringido praticamente às ciências do cérebro.

Outra observação importante $\varepsilon$ que isso indica um alheamento crescente da pesquisa clínica, face ao contexto da vida dos pacientes. Isto é, essa pesçuisa refletiria o trabalho clínico cada vez mais especializado, num percurso em que há um afunilamento na seleção do fenômeno observado. Em 1946 os clínicos gerais viam a pesssoa inteira e, para muitos, o corp. ia além da pele, englobando também o corpo familíar e social. Nas décadas posteriores, o olhar do clínico foi se afunilando, num processo focalizante, de cada vez mais observar apenas um detalhe. Do corpo social foi se reduzindo à observação do corpo até a da pele, depois de um órgão, de cêlulas, dos cromossomos e, enfim, de apenas um gen. 

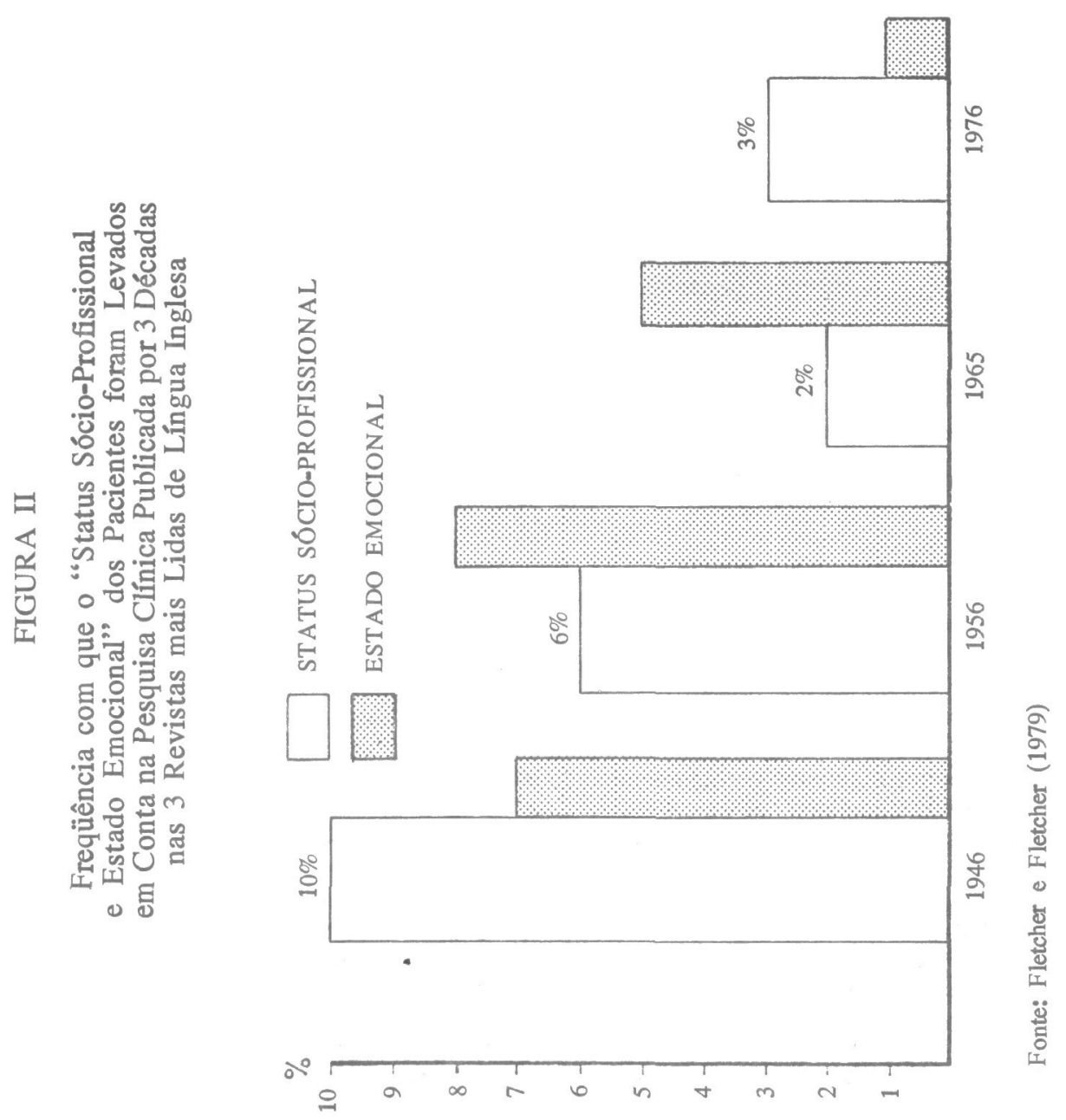
Também é importante comentar que a diferença na valorização dos fatores sócio-econômicos por epidemiologistas e clínicos foi crescente, com o risco de se formar um abismo entre um e outro, com absoluta impossibilidade de um trabalho integrado pelos dois grupos. Esse abismo é de suma importância, com um mútur isolamento e exclusão progressivos.

\section{PERSPECTIVAS E AVANÇOS A CURTO PRAZO DA EPIDEMIOLOGIA}

Tudo indica que o mais promissor avanço da epidemiologia é sua aplicação para ordenar e interpretar dados da Antropologia, o que foi bem delineado recentemente por Sperber (6). Uma nova moda que "pega" e logo desaparece e certos traços culturais que permanecem quase indefinidamente näo diferem de epidemia e nível endêmico, respectivamente. A distribuição geográfica, cronológica e por atributos de pessoa dos mais diferentes dados empíricos colhidos pelo antropologista contribui muito para conhecer melhor os fenômenos dessa área. Para Sperber, o mais fundamental é que a epidemiologia constitui importante altemativa para compreender as "transformaçōes" que sofrem os fenômenos culturais, enfim, uma nova via de acesso ao que ocorre na aculturação. Destaca-se que tal uso nāo compreeende a noção de "dano na população", isto é, não há incorporação da vertente oriunda dá patologia; o que Sperber mostrou foi o lado da inteligibilidade do método para constituir uma "epidemiologia das representaçōes" dos fenômenos da Antropologia.

A aplicação do método epidemiológico aos dados gerados no meio hospitalar e ambulatorial tem tido, recentemente, um progresso enorme nos países desenvolvidos. Vandenbroucke (8) descreve-a como sendo uma "nova ciência", com um objeto particular, no qual a produçîo científica aumenta muito, com novas revistas, cursos etc. No Brasil observa-se o surgimento de novos serviços em hospitais, que se inserem nessa assim chamada epidemiologia clínica. Contudo, como acentua Terris (7), às possibilidades de aplicação da epidemiologia são muitas, as quais vão, pouco a pouco, sendo redescobertas, como é o caso da aplicação na clínica. Esse autor enfatiza que todos os grupos populacionais pode!n ser classificados de acordo com níveis de exposição a riscos, os quais são muito reais para serem deixados de lado como se não fossem práticos. As atividades de saúde pública não têm feito valer a noção de risco; esse conceito é a mais importante conquista teórica da epidemiologia, que visa um fim dos mais práticos, mas que talvez tenha que ser redescoberto. 
Some new approaches on the use of epidemiology were concisely reviewed in this paper. Firstly, the importance of the health consequences of socio-economic factors, which have increased in epidemiologic research, and decreased too much in studies from the clinical research area. The population's health state evaluation was then discussed, when was remarked that without operational indicators of positive health that evaluation is necessarily limited. Attention was also paid to progress in methods, from which two epidemiologic approaches have been developed. In one of them rigour in methods and in data analysis by computer are endlessly searched for, while health solve-problem is focused in the other. Therefore, epidemiology cannot be separated from social, economic and behavior sciences, which altogether may achieve that goal.

\section{REFERÊNCIAS BIBLIOGRÁFICAS}

1. CASTIEL, L.D. (1989). Freud - um epidemiologista? Cad. Salide Pablica., 4: 316, 325.

2. FLETCHER, R.H. and FLETCHER, S. W. (1979). Clinical research in General Medical Journals, New Eng. J. Med., 301: $180-183$.

3. GOLDBERG, M. (1982). Cet Obscur Objet de l'Épidémiologie. Sciences Sociales et Sante, n: 1: 55-110.

4. REID, D, D. (1948). Sickness and stress in operational Flying. Brit J. Soc. Med., 2: 123-131.

5. SAWYER, D. (1986). The Potential Contribution of Social Research to Control of Malaria in Brazil. Mem. Inst. Oswaldo Cruz, 81 (Suppl. II): 31-37, 1986.

6. SPERBER, D. (1985). Anthropology and Epidemiology: Towards an Epidemiology of Representations, Marr, 20:73-89.

7. TERRIS, M. (1980). Epidemiology as a Guide to Health Policy. Ann. Rev. Publ. Health, 1: 323-344.

8. VANDENBROUCKE, J.P. (1989). On the New Clinical fashion in epidemiology. Epidem. Infection, 102: 191-198. 